\title{
Une structure dialogique et plastique : une hypothèse de l'usage de Néopass@ction
}

A dialogical and plastic structure: hypothesis for the use of Neopass@ction

\section{Laurence Espinassy}

\section{(2) OpenEdition}

\section{Journals}

Édition électronique

URL : http://journals.openedition.org/activites/2863

DOI : $10.4000 /$ activites. 2863

ISSN : 1765-2723

Éditeur

ARPACT - Association Recherches et Pratiques sur les ACTivités

Référence électronique

Laurence Espinassy, «Une structure dialogique et plastique : une hypothèse de l'usage

de Néopass@ction », Activités [En ligne], 13-2 | 2016, mis en ligne le 15 octobre 2016, consulté le 01

mai 2019. URL : http://journals.openedition.org/activites/2863 ; DOI : 10.4000/activites.2863

Ce document a été généré automatiquement le 1 mai 2019.

\section{c) (i) $(\Theta$}

Activités est mis à disposition selon les termes de la licence Creative Commons Attribution - Pas d'Utilisation Commerciale - Pas de Modification 4.0 International. 


\title{
Une structure dialogique et plastique : une hypothèse de l'usage de Néopass@ction
}

\author{
A dialogical and plastic structure: hypothesis for the use of Neopass@ction
}

\author{
Laurence Espinassy
}

\section{NOTE DE L'ÉDITEUR}

Article soumis le 31/01/2016, accepté le 30/06/2016

1 Le questionnement qui préside à cet article naît du constat suivant : lors de la conception d'un artefact destiné à la formation des enseignants, la structure organisationnelle et la forme visuelle finale s'avèrent proches d'outils culturels connus. Notre propre formation, doublement orientée entre les arts plastiques et l'analyse ergonomique de l'activité des professionnels de l'Education, nous fait porter un regard attentif à la structure formelle de cet artefact, à la fois visible et transparente.

2 L'hypothèse théorique soutenue dans cet article est : 1 / La structure de cet artefact de formation a été conçue pour créer un milieu propice au développement professionnel, en ouvrant l'espace d'un milieu dialogique favorable à la mise en récit de l'histoire et de l'expérience professionnelle de chacun. 2 / En termes d'usage, collectif ou individuel, l'héritage historico-culturel de l'approche de certaines formes artistiques favoriserait l'appropriation des situations professionnelles proposées par cette structure hybride, en se rattachant à des systèmes de lecture « déjà-là » et stabilisés.

3 Notre réflexion s’inscrit dans le cadre général qui suit. D’une part, Rabardel (1995, p. 183) postule que dans un usage habituel, l'artefact, en tant que moyen, est « transparent ", car l'utilisateur n'a nul besoin d'en avoir une connaissance consciente pour en faire usage selon des schèmes d'utilisation associés, résultant d'une construction propre du sujet, ou d'une appropriation de schèmes sociaux d'utilisation déjà formés extérieurement à lui. 
D'autre part, Vygotski nous invite à placer autrui, les situations d'interaction ainsi que les outils culturels qui y sont mis en œuvre (dont le langage), au cœur même d'une théorie du développement humain. Par ailleurs, Cole, Hakkarainen et Bredikyte (2010) apportent les précisions suivantes : la culture correspond aux connaissances, aux outils et aux attitudes accumulés au fil des générations qui imprègnent le milieu immédiat de l'enfant, y compris les «pratiques » culturelles des membres de la famille nucléaire et des proches. L'apprentissage est vu comme la modification relativement durable du comportement ou de la compréhension résultant de l'expérience de l'enfant. Le développement se traduit par des changements qualitatifs dans l'organisation fonctionnelle interne du cerveau, du corps et du comportement de l'enfant ainsi que par des modifications dans la relation entre l'enfant et l'organisation de ses expériences socioculturelles. La culture joue un rôle capital dans la façon dont les enfants interprètent le monde. Ces auteurs rejoignent les thèses de la psychologie culturelle et la pensée de Bruner (1991) : la culture donne forme à l'esprit.

4 Penchons-nous sur l'artefact qui nous intéresse ici. Pour résumer, car nous y reviendrons en détail, Néopass@ction est un outil de formation qui offre des ressources réalisées à partir de travaux de recherche fondés sur l'observation du travail des enseignants. Il se présente en plusieurs grands thèmes définis selon les difficultés majeures rencontrées par les professeurs débutants (l'entrée en classe, l'aide aux élèves...). Chaque thème est traité par une équipe universitaire, mais pour conserver une unité de conception d'ensemble, toutes doivent respecter une charte commune qui énonce les principes suivants :

«- Partir prioritairement des situations vécues par les débutants en mobilisant secondairement l'activité d'enseignants plus expérimentés ;

- Partir systématiquement des enjeux liés à l'activité professionnelle... ;

- Associer systématiquement aux extraits vidéo de classe la mise en mots des vécus professionnels pour en comprendre le sens, l'efficience, les aspects spécifiques et typiques, etc. ;

- Recenser, décrire, analyser les modalités d'enseignement recueillies et en interroger la pertinence et l'efficacité attendue, supposée ;

- Proposer systématiquement pour chaque situation professionnelle le regard croisé d'enseignants débutants, d'enseignants expérimentés et de chercheurs pour montrer la complexité du travail enseignant et sa dynamique de transformation ;

- Organiser..., des retours d'expérience sur l'utilisation de ces ressources » (Ria \& Leblanc 2011, p. 162).

5 Nous avons été en charge de la conception du thème 2 « aider les élèves à travailler et à apprendre», dont l'intitulé reprend une prescription officielle du référentiel de compétences des enseignants.

6 Les thèmes s'affichent principalement à l'écran sous forme de vignettes photographiques légendées (photogrammes de brefs extraits d'enregistrements vidéo de situations de travail), que l'usager doit activer pour accéder au déroulement de l'activité filmée d'autrui. On est donc en présence d'un matériau riche, de ressources constituées d'une multiplicité de documents qui comprennent aussi bien des images fixes, des vidéos et des textes produits par différents acteurs alliés au processus de conception, résultant d'un travail engagé dans et par plusieurs collectifs.

7 On peut craindre que cette complexité soit davantage une difficulté plutôt qu'une aide pour l'utilisateur. Comment une telle plateforme peut-elle être un instrument potentiel de médiation, de compréhension et de développement de l'activité enseignante? Comment en faire usage? 
Il s'agit ici de développer un point de vue plus globalisant en complément des études déjà publiées à propos de Néopass@ction dans cette revue (Ria \& Leblanc, 2011; Ria \& VidalGomel 2014, Activités, 11(2), «Conception d'environnements de formation : une entrée par l'analyse de l'activité »), ou dans Recherche et formation, 75 | 2014 (Analyse de l'activité des enseignants débutants et vidéoformation, Saussez, 2014b). Ces publications, ou encore les résultats apportés par une thèse récente (Flandin,2015), portent sur des données empiriques permettant d'évaluer de manière quantitative et qualitative l'usage de cette plateforme en ligne, que nous ne trouverons pas ici.

9 Nous abordons une autre dimension de cette plateforme : en conclusion du numéro 11-2 d'Activités, Saussez (2014a) invite à « continuer à problématiser la question du langage, des instruments et de l'inscription sociohistorique des environnements de formation » dans leurs apports à la « construction du rapport au métier ». C'est à cette invitation que nous proposons de répondre, en interrogeant la nature et la forme de cet objet intermédiaire, médiateur entre l'activité passée des uns (dont on voit des traces filmées) et celle à venir des autres (usagers, professeurs débutants et formateurs).

Dans une première partie, nous rappelons quelques éléments du cadre conceptuel et méthodologique à partir duquel nous avons conçu cet artefact de formation, en détaillant les hypothèses instrumentales, et le choix d'une entrée par les «dilemmes professionnels » (Prot, 2011) pour faciliter la compréhension du travail d'autrui, qui ont présidé à la constitution du matériau filmique. Puis, revenant sur la conception du thème 2 de la plateforme et en nous attachant à l'organisation visuelle de ses écrans, nous donnons un exemple de la formalisation choisie. Enfin, nous questionnons le processus de dynamique des émotions, son lien à l'action, ainsi qu'à l'activité de transformation de l'expérience de l'autre, suscités par la confrontation de l'usager à ces extraits vidéo de l'activité d'autrui.

11 Nous formulons l'hypothèse que, si l'environnement proposé par cet artefact de formation est susceptible de créer un milieu propice au développement professionnel, c'est en particulier grâce à sa forme et sa structure, conçues comme « un système suffisamment souple, suffisamment plastique pour laisser des degrés de liberté à l'activité en situation...» (Béguin, 2013, p. 149), qui contribuent à la spécification d'« espaces d'activité future" ou possible (Daniellou, 2004). Les écrans polymorphes et polydimensionnels de Néopass@ction, de nature visuelle, permettent d'activer, au choix, de multiples extraits vidéo identifiables par des «mots du travail ». Nous soutenons que leur agencement crée, non seulement des mises en relations entre les diverses composantes du métier enseignant, mais encore, l'espace d'un milieu dialogique propice à la mise en récit de l'histoire et de l'expérience professionnelle de chacun.

12 La seconde partie s'intéresse à la matérialité de l'artefact et à l'espace ouvert par les écrans du thème 2 de Néopass@ction. Nous avançons que la structure visuelle et lisible choisie pour didactiser cette ressource (Félix \& Espinassy, 2011) reprend à son compte certains aspects du fonctionnement d'œuvres plastiques et littéraires, correspondant à des formes textuelles, intertextuelles, visuelles ou participatives historiquement et culturellement fondées. Nous croisons ici des réflexions empruntées à la théorie de l'activité langagière - issue initialement de l'analyse de l'activité de travail et soutenue par Daniel Faïta (2007, 2011, 2013a) -, et d'autres, issues des sciences de l'art. Il s'agit de domaines rarement confrontés; pourtant, les relations fond-forme, intentionnalitéstructure, complexité-lisibilité, hétérogénéité-cohérence..., sont autant de 
préoccupations communes aux artistes, qu'à tous ceux qui cherchent à donner accès à une production de sens à partir d'unités de significations.

Nous soutenons, que c'est bien parce que des codes d'organisation spatio-temporelle socialement construits sont familiers aux usagers, qu'ils leur permettent d'accéder, sans se perdre, aux juxtapositions d'images, de textes, de films de Néopass@ction, et d'accéder partiellement à la complexité de l'activité d'autrui.

Pour conclure, nous mettrons en discussion l'usage potentiel de ces ressources en formation professionnelle.

\section{La conception d'un artefact à vocation instrumentale}

15 Ria et Leblanc exposant les principes généraux qui ont présidé à la conception de cette plateforme, précisent que «l'objet central de formation dans la ressource n'est pas pensé en termes de structures constituées et de contenus prédéterminés, mais plutôt en termes de processus d'individuation, de "procès créatif" défini comme un processus d'objectivation de l'expérience, de la relation à soi-même et au monde extérieur » (2011, p. 154). Dans ce cadre commun, on constate des variations liées aux choix théoriques et méthodologiques sous-jacents à chaque équipe universitaire en charge d'un thème ${ }^{1}$.

\subsection{Parti pris théorique et méthodologique}

Voici quelques éléments du contexte et du cadre théorique et méthodologique que nous avons adopté, déjà décrits par Félix (2014) et Félix et Espinassy (2012). En résumé, la conception s'organise autour de situations de travail problématiques, progressivement dégagées lors d'interventions réalisées sur plusieurs années auprès de professeurs débutants et confirmés. Ces propositions résultent d'une expérience commune qui sert de point de départ pour le développement d'une autre expérience collective.

17 Toutes les situations de travail filmées qui composent ce thème résultent des interventions que nous avons conduites au sein des établissements scolaires et au plus près des professionnels qui nous ont sollicitées pour les seconder dans la prise en main de situations nouvelles de travail, toutes supposées mettre en œuvre les prescriptions d'individualisation et d'autonomisation de l'aide à apporter aux élèves dans leurs apprentissages scolaires (Félix \& Saujat, 2008 ; Félix, Saujat, \& Combes, 2012).

En cohérence avec le cadre théorique de l'ergonomie de l'activité selon une approche clinique (Saujat, 2010, Faïta \& Saujat, 2010), et celui du cadre méthodologique de l'autoconfrontation (Faïta \& Veira, 2003, Clot \& Faïta, 2000), nous avons cherché à mettre les professionnels en situation d'analyse de leur activité dans une perspective de transformation des situations de travail. Dans une lignée Vygotskienne de la conscience, l'action matérielle reconstruite verbalement dans l'interaction sociale sur le plan de la conscience se transforme en une action qui s'organise sur de nouvelles bases. C'est en cela que la conscience est considérée comme "l'expérience vécue d'une expérience vécue " (Vygotski, 1925/1994, p. 78); un processus de «traduction d'une activité en une autre activité, [une] liaison entre activités» (Clot, 2003, p.12). Dans notre cadre méthodologique, la transition d'une activité première (vidéoscopée) en une activité à venir est rendue possible par la relation avec une troisième: l'activité dialogique 
proposée. Elle représente en quelque sorte ce que Vygotski nomme le «contact social avec soi-même » (1925/1994, p. 91)».

Précisons ce que nous entendons par «dialogisme» en référence aux thèses bakhtiniennes, qui sera utilisé souvent par la suite. Nous reprenons la note de synthèse de Saujat (2010) qui souligne que selon François (1996), "parler de "dialogisme" est ici synonyme d"interprétation" [...] au sens de ce qui rend capable de faire entrer un texte ou un objet signifiant en général dans des ensembles où ils font sens » (p. 13). François précise que texte ne signifie pas ici texte écrit, mais désigne « un énoncé ou un ensemble d'énoncés [...] en tant qu'on le prend comme objet de réception (de lecture si on veut) dans sa triple relation aux autres textes, à notre compréhension responsive, aux effets de mise en mots dont il est capable» (p. 14). Ces effets se produisent dans un espace de jeu où il $\mathrm{y}$ a de l'ouvert, de l'inattendu, espace justiciable d'une linguistique de l'événement en quête de la diversité des façons de signifier qui se manifeste dans la circulation du sens, des distances entre interlocuteurs (François, 1993). On ajoutera « qu'au-delà encore, c'est la multiplicité des voix qui se manifeste dans les énoncés de chacun : voix de l'autre, mais aussi du collectif, de l'organisation, ainsi que [le] retravail des énoncés du sujet par des valeurs d'arrière-plan » (Faïta, 1999, p. 136).

\subsection{De l'intervention-recherche à la formation, une forme de didactisation}

Notre statut conjoint d'intervenant-chercheur et de responsable de formation d'enseignants nous a poussés à allier progressivement des logiques initialement distinctes: celles de l'intervention, de la recherche, puis celle de la formation, pour concevoir cet artefact (Félix, 2014). Dans ce processus, nous avons mobilisé différents collectifs de travail (enseignants novices, enseignants chevronnés, formateurs, formateurs de formateurs...) selon une entrée par les difficultés du métier, considérant avec Wisner que le travail consiste moins à appliquer des prescriptions -ici essentiellement d'individualisation de l'aide aux élèves - qu'à vaincre des difficultés du métier (Wisner, 1995).

La construction du thème 2 résulte donc d'un travail engagé dans et par plusieurs collectifs distincts, de travail et de formation, exerçant dans des milieux différents, et que nous retrouverons plus loin dans la description de la structure du thème. Ce matériau dense, composé par un ensemble de documents résultants des divers enregistrements vidéo témoigne des multiples activités qui se déploient dans ces différents espaces-temps construits dans le cadre du dispositif d'intervention, retravaillées au fil de nos interventions dans les établissements scolaires qui en font la demande. Contrairement à une production cinématographique, il n'y a pas de scénario initial, ni de montage d'extraits vidéo les uns avec les autres dans une intentionnalité de récit. La sélection des situations de travail visibles dans la plateforme résulte de leurs mises ou remises à l'épreuve systématique dans et par différents collectifs de travail ; ce matériau constitue la base que nous avons eu à didactiser (Félix \& Espinassy, 2012).

Comment ne pas se perdre dans cette multiplicité de ressources? Comment rendre intelligible cette masse de sons et d'images extraits du monde du travail enseignant, qui, selon Wolff, « comme tout matériau indéfini, ne peuvent rien signifier d'autre que ce dont ils témoignent »? (2015, p. 54). Selon cet auteur (dont la réflexion est centrée sur la musique et à qui nous empruntons pour établir des parallèles avec d'autres domaines 
d'activités), c'est par « réduction» et «mise en ordre " que «le monde musical a été obtenu pour s'opposer à l'univers sonore », et que le propre de la musique, est de " nous avoir fait passer de l'univers chaotique des sons à un monde peuplé d'individus en relation possible les uns avec les autres", en produisant "des éléments discontinus servant ensuite à reformer une continuité significative » (ibid., p. 85).

Nous témoignons donc ici de la réduction et de la mise en ordre opérées dans la construction de notre thème afin de faire dialoguer entre eux les différents niveaux d'expertise professionnelle présents, et de constituer comme ressource pour d'autres professionnels cette arène de conflits de critères et de débats professionnels, entre gens de métier. Dans une visée de transmission-appropriation de contenus culturels élaborés initialement à l'extérieur du sujet, des choix structurels et organisationnels ont été faits pour permettre à l'usager d'entrer dans ce mouvement de tension entre le subjectif et le social, entre le sens et l'objet de l'activité qui, pour reprendre les mots de Léontiev « fait de la conscience ce qu'elle est : un développement possible ou impossible de l'activité du sujet » (Leontiev, 1975/1984, p. 108) (Félix, Amigues, \& Espinassy, 2014). Selon Brossard (2012, p. 104), dans le cadre scolaire, le "travail didactique » a pour objectif de transformer ce qui a été produit lors d'un travail déjà effectué dans les domaines d'expériences antérieurs et extérieurs à l'école. Les didactiques lui semblent être le terrain d'élection où la théorie du développement humain élaborée par Vygotski devrait trouver son lieu d'opérationnalisation, car pour ce dernier, l'enseignement n'est en rien un travail d'inculcation : on n'implante rien.

Il nous a donc fallu opter pour une organisation de l'ensemble des ressources afin que l'espace didactique proposé devienne le lieu de rencontre et de mise en tension du travail visible des uns et de celui de l'usager dans ses différents domaines d'expérience.

\subsection{Adaptation Fond / Forme / Intentionnalité}

Les différentes phases d'intervention-recherche au sein de ces divers collectifs de travail sont visibles dans cet environnement de formation. L'utilisateur est soumis à un écran où les situations sont organisées selon une logique qui part des difficultés que les enseignants disent rencontrer au quotidien, et des alternatives auxquelles ils sont confrontés pour trouver des solutions aux problèmes qui se posent à eux en matière d'aide et d'accompagnement des élèves. Rappelons que chacun des éléments filmiques constituant la ressource, ainsi que leur agencement, ont été soumis à chacune des étapes de la conception à l'approbation des différents collectifs et acteurs décrits précédemment. C'est donc à la mesure du développement historique des rapports entre ces derniers et les concepteurs que s'est développé l'instrument. Il s'agit donc "d'un processus dont la structure est dialogique : le résultat du travail de l'un est remis en travail dans l'activité de l'autre, donnant lieu à une réponse » (Béguin, 2013, p. 152).

Notons qu'une structure de base distribuant l'ensemble des vignettes visibles, et correspondant aux contraintes informatiques, est imposée à tous les thèmes de Néopass@ction. Mais alors que l'on trouve dans la vignette centrale du thème 1 une situation de classe qui sera commentée par le «vécu professionnel» de la personne filmée (présent dans une vignette secondaire), c'est un choix inverse qui a été retenu dans le thème 2. C'est l'extrait d'autoconfrontation simple qui est mise en avant, et par là même le-les dilemme(s) auxquel(s) est confronté le débutant, la situation de classe passant au second plan. Pour le professionnel qui l'affronte, un dilemme de métier 
propose une alternative de solutions, également insatisfaisantes, parmi lesquelles il devra arbitrer afin d'agir. Selon Prot (2011), ces dilemmes appartiennent au patrimoine générique du métier et l'obstacle qu'ils constituent, ainsi que les diverses solutions qui permettent de les " gérer", sans les "régler ", sont connus des professionnels. Le cadre méthodologique des autoconfrontations et les dialogues qu'il génère, permet aux professionnels mobilisés ici dans divers collectifs de les redécouvrir, de les reconsidérer, d'en débattre.

Voici, par exemple, comment se présente la déclinaison du thème «Aider les élèves à apprendre et à travailler " selon l'entrée "Montrer ou faire découvrir » (dans la frise horizontale en haut) incarnée par Claire, dans une classe de mathématiques en $3^{\mathrm{e}}$. On y trouve :

Figure 1 : Organisation du thème " aider les élèves à travailler et à apprendre » - Entrée : " montrer ou faire découvrir » (http://neo.ens-lyon.fr/neopass/index.php?themes=2\&activites=12).

Figure 1: Organization of the section entitled "Helping pupils to work and learn". Current selection: "Showing versus allowing discovery"

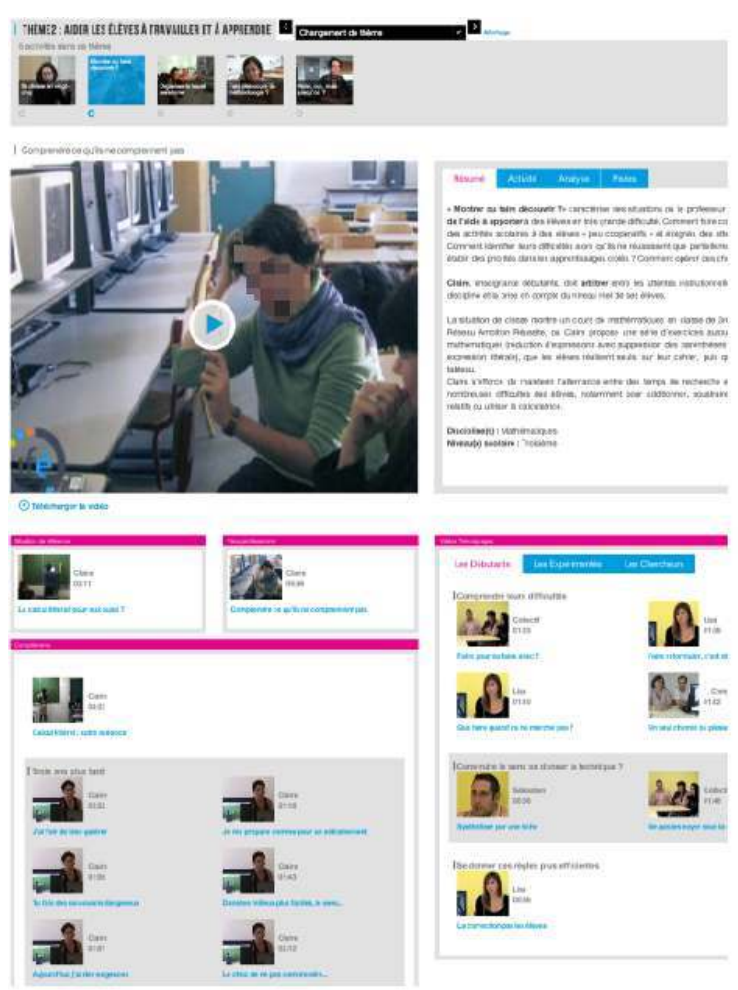

(en raison de la difficulté à reproduire ici la lisibilité de l'écran, nous invitons le lecteur à consulter la page du site).

1. Des déclinaisons illustrées par cinq vignettes horizontalement exposées en haut de l'écran, proposant des situations professionnelles de trois professeures néotitulaires, Guillemette, Claire et Albane. Chacune des situations retenues se présente en dessous, au travers du travail de chacune d'elles, comme ici pour Claire. On constate, que pour ces professeures, « Aider les élèves à travailler et à apprendre » se pose, plus particulièrement, du point de vue de l'individualisation de l'aide, de ses modalités et contenus susceptibles d'encourager l'autonomie, de l'encadrement du travail personnel des élèves hors de la classe et de sa place en retour dans l'avancée du cours en classe, des enjeux éducatifs, politiques, et sociaux de l'aide. En termes de métier, puisque ce sont leurs formulations de professionnelles qui ont été retenues, cela se traduit par les titres des vignettes de la frise du haut: "Se diviser 
en 25 », « Montrer ou découvrir », " Organiser le travail personnel », « Faire des cours de méthodologie », "Aider, oui, mais jusqu'où? ». Cette frise est composée de situations issues du travail conjoint des collectifs associés à la recherche.

2. Des vignettes placées dans la partie inférieure de l'écran et qui montrent des moments différents de la carrière de ces trois enseignantes débutantes. Diverses séquences donnent ainsi à voir des préoccupations qui se transforment, se recyclent au fil des années. Ce différentiel d'expérience professionnelle, à travers les efforts que déploient ces trois jeunes enseignantes pour organiser leur travail en même temps que celui de leurs élèves est objet de réactions, de discussions, parfois de débats controversés que l'on retrouve dans :

3. Visibles dans la zone centrale à droite de l'écran (sélection au choix) :

- les propos des professeurs stagiaires qui disent se reconnaître - ou pas - dans les pratiques de leurs collègues débutantes (Guillemette, Claire et Albane), et qui tentent d'interpréter, à l'aune de leurs propres préoccupations, leurs actions et les motifs qui orientent celles de leurs collègues dans l'exercice de leur métier.

- les propos des enseignants chevronnés, toujours soumis à de difficiles négociations et de vifs débats, jamais tranchés et qui les « travaillent » toujours.

\section{4. Émotions, activité et développement}

29 Ce sont donc différentes facettes du métier et différentes manières de concevoir l'aide aux élèves que nous donnons à lire et à mettre en discussion dans ce thème 2 , qui témoignent tout autant des convergences de pensée et de pratiques, que des conflits de critères et de valeurs, inhérents au métier enseignant. En d'autres termes, ce que nous avons tenté de scénariser dans le cadre contraint d'un environnement numérique, ce sont, tout à la fois, des difficultés génériques (reconnues comme telles par divers collectifs professionnels) et les diverses réponses qu'apporte le métier aux dilemmes historiques et impersonnels soulevés par ces situations.

Pour autant, ces dilemmes et les situations qu'ils mettent en jeu, bien que caractéristiques de l'exercice du métier, ne sont pas directement accessibles aux professionnels, et encore moins à des étudiants qui n'auraient pas participé au dispositif d'intervention-recherche qui est le nôtre, mais à qui ces ressources sont "adressées ». Débarrassées de leurs conditions d'exercice, les situations de classe présentées sont très courtes et peu loquaces; de même que les gestes de l'aide aux élèves, souvent indicibles, sont difficilement repérables à première vue et en dehors de toute construction intentionnelle pour les identifier et se les approprier.

31 Comment faire alors pour que les professionnels débutants s'y intéressent, se les approprient, et transforment ces ressources en instrument de leur activité ?

Nous soutenons que la conception de l'artefact, rend non seulement visible les dilemmes de métier, mais que de plus, suite à la déstabilisation émotionnelle produite chez des usagers face à la complexité du métier, sa structure permet d'organiser des débats à ce sujet. À nos yeux (Félix, Amigues, \& Espinassy, 2014), l'activité artefactuelle considérée comme relation entre les hommes n'est pas faite que de consensus ou de compréhension mutuelle; elle est surtout faite d'incompréhensions, de divergences, de malentendus. Si l'activité est un trait d'union, c'est « l'unité des contraires » et « leur interprétation » qui relient les interlocuteurs (Sève, 2002). Comme le note Bronckart « les apports externes ne sont générateurs de développement que dans la mesure où les conflits qu'ils engendrent sont "traitables" par la personne » (2008, p. 240). 

continuité marquent l'interaction individu-milieu. C'est ce qui se passe lorsqu'une variation se produit du côté du milieu et que l'individu ne dispose pas des structures de connaissance et d'action nécessaire pour y répondre. C'est également ce qui se produit quand une variation intervient du côté de l'individu (besoins, désirs, aspirations...) et que le milieu ne fournit pas les éléments que celui-ci attend en réponse à cette variation ». L'auteur souligne que le partage social des émotions «implique deux éléments: la réévocation de l'émotion sous forme d'un langage socialement partagé, et la présence, au moins à un niveau symbolique, d'un partenaire auquel cette réévocation est adressée " (Rimé, 2005, p. 86). À nos yeux, Néopass@ction présente cette variabilité, à la fois des conditions de travail en milieu enseignant, et celle des ressources de métier mises en débat par les professionnels. L'usager débutant ne disposant pas des structures de connaissance et d'action nécessaire pour y faire face, doit trouver des interlocuteurs et des formes pour parler, notamment dans le cadre de sa formation, de «ce qu'il peut se passer - de ce qu'il s'est passé - de ce qu'il va se passer ", car selon Wolff, "cette opposition de la tension de l'être vivant face aux événements inattendus et de la détente face aux événements attendus ou au repos du monde, est fondatrice de la plupart de nos émotions » (2015, p. 32). (1925/2005, p. 18), et Rickenmann (2012, p. 318) souligne qu'ainsi, « il évoque la notion de travail en tant qu'activité de transformation matérielle et symbolique de l'environnement ». Que ce soit dans le domaine artistique ou dans celui du monde du travail, la modification d'un matériau en vue de créer des formes implique que les actions (plastiques ou autres) et leur produit s'insèrent surtout dans une activité dont le but est la trans-formation de l'expérience de l'autre. L'auteur poursuit :

«travailler et "donner forme au sentiment" ne consiste pas à "traduire" matériellement (production) ni à "réveiller" (réception) quelque chose qui serait à l'intérieur des sujets, mais à mettre en œuvre un dispositif technique et pragmatique modifiant les rapports corporels au monde sensible et la manière dont il nous affecte. Historiquement et culturellement définie, cette "technique" est une cristallisation, sous forme d'objets et d'activités, des modes de résolution des rapports humains avec l'environnement. Les deux versants, celui de l'objet et celui du corps agissant, se co-définissent dans la forme comme activité ».

D'une part, Vygotski insiste sur l'importance de la dimension sémiotique de ce processus qui transforme l'objet culturel, et l'œuvre d'art en particulier, en véritable outil de développement de ces processus de co-définition (1934/1997), et d'autre part, il ne conçoit la dynamique des émotions que fondamentalement liée à l'action (1933/1998). Du point de vue pragmatique, cette unité de l'affect, qui se manifeste au niveau de la corporéité de l'existence, « fait signe » et participe aux systèmes de significations à partir desquels émerge le sens de nos rapports au monde. Les émotions ne sont pas des réflexes découlant d'un rapport objet vers sujet; ce sont des ré-actions qui s'inscrivent dans un processus évolutif d'interprétation de ce qui fait signe pour le sujet dans la situation et qui oriente son agir (Rickenmann 2012, p. 319). Wolff le dit autrement :

« Toute émotion est causée dans un sujet par des événements qui lui arrivent et qui l'affectent. S'il ne lui arrive rien, il ne ressent rien... L'émotion, c'est l'effet subjectif et présent d'un événement présent, passé ou à venir... Autrement dit, ça nous fait quelque chose, psychiquement et physiquement. Selon ce qui nous arrive, nous ressentons des émotions particulières, qualifiées selon l'événement, et affectées positivement ou négativement; et nous éprouvons aussi des effets physiques 
éventuellement accompagnés de mouvements orientés positivement (attraction vers ceci) ou négativement (fuite loin de cela)» (2015, p. 100-101). spécificité de sa forme, crée un milieu propice au développement professionnel; ce processus s'institue notamment à partir du vide laissé entre les vignettes photographiques, les extraits vidéo et les registres écrits visibles sur les écrans de Néopass@ction. Cet espace vacant implique que l'usager doive effectuer un choix entre deux vignettes ; nous le rapprochons de la réflexion de Brossard (1989) à propos du sujet construisant un «espace mental» devenant le lieu des activités de résolution de problème : sélection de l'information, représentation du but et des sous-buts, choix de procédures, etc. Cet espace mental est donc, de par son origine, un espace social. S'inspirant des travaux de Bakhtine, J. Wertsch s'efforce d'élargir sur ce point l'apport de Vygotski, pour qui le sens peut ne pas être lié au seul contexte immédiat, mais peut être en relation avec des contextes sociaux plus larges (Wertsch, 1985). L'espace mental, de ce fait, n'est pas un espace neutre, mais un lieu chargé de sens. L'espace vacant entre les différents registres visibles référant au travail d'autrui peut laisser par exemple place à l'évocation des expériences vécues lors des stages professionnels par les professeurs novices usagers de la plateforme et offrir l'occasion de la reconstruction pour soi d'une situation discursive. Ce vide pourrait être un des lieux propices au développement de l'activité professionnelle. Il correspond à la conception même de cet artefact, comme « un système suffisamment souple, suffisamment plastique pour laisser des degrés de liberté à l'activité en situation... » (Béguin, 2013, p. 149), contribuant à la spécification d'« espaces d'activité future » ou possible Daniellou (2004). Les propos tenus face la plateforme par Christelle, professeure stagiaire, témoignent de l'investissement possible de cet espace :

"Quand on voit ces vidéos, on se dit "ben moi j'aurais fait ça" parce qu'on fait toujours référence à sa classe et aux élèves que l'on connait... Et puis, là, on réagit à froid; eux, ils sont dans l'ambiance, dans la situation, c'est pas facile, on a pas forcément les bonnes réactions au bon moment. Après avec recul, on se dit "ah ben si j'avais su, j'aurais fait ça", et c'est ce qui se passe aujourd'hui devant cet écran : on observe quelqu'un en se disant "ben moi j'étais dans la même situation, j'ai peutêtre fait ça, mais je ne m'en suis pas rendue compte...", c'est intéressant ».

Mais le développement ne se décrète pas: l'idée même de "transmettre " induit l'existence des ressources nécessaires à l'acte de transformer autrui (Faïta, 2012). Les écrans polymorphes et polydimensionnels de Néopass@ction, de nature visuelle, permettent d'activer au choix, de multiples extraits vidéo; mais encore faut-il que l'usager entre en action et dans l'exploration de la plateforme.

Nous souhaitons montrer ici comment la structure même de cet artefact crée un espace susceptible de provoquer des mises en relations entre les diverses composantes du métier ainsi qu'un milieu dialogique propice à la mise en mots de l'histoire et de l'expérience professionnelle de chacun. Cette visée fait écho aux thèses bakhtiniennes selon lesquelles les « mots » de mon discours ne font « sens » pour moi qu'en relation (opposition) à ceux des autres.

Pour ce faire, nous voulons tenter un rapprochement entre ce type de production artefactuelle et le fonctionnement perceptif et participatif induit par certaines autres productions, notamment artistiques. 


\section{De la matérialité de l'artefact à l'élaboration d'espaces professionnels critiques}

39 Nous croisons ici des réflexions empruntées à la théorie de l'activité langagière - issue initialement de l'analyse de l'activité de travail et soutenue par Daniel Faïta (2007, 2011, 2013b) -, et d'autres, issues des sciences de l'art. Dans le cadre de notre réflexion, se rapprochement s'explique par :

- La convergence d'intérêt autour de la production discursive sur le travail, y compris artistique ; en effet, les sciences de l'art s'intéressent aux œuvres d'au moins deux façons en général : par le « discours poïétique » s'intéressant à la genèse de la création artistique, et le « discours esthétique » qui analyse la manière dont les œuvres s'adressent au spectateur selon les choix opérés par l'artiste.

- Le fait qu'activités de travail et activités artistiques sont des domaines rarement confrontés, car on éloigne le plus souvent les activités industrieuses et celles liées aux champs créatifs, tant subsiste l'idée romantique que la conception artistique tiendrait du « génie » ou de la pure «sensibilité » de l'individu, déconnectée des conditions matérielles de sa réalisation. Pourtant, les relations fond-forme, intentionnalité-structure, complexité-lisibilité, hétérogénéité-cohérence..., sont autant de préoccupations communes aux artistes, et à tous ceux qui cherchent à donner accès à une production de sens à partir d'unités de significations.

- La proposition de Vygotski d'élaborer une explication matérialiste des émotions ; il soutient que l'émotion esthétique est une émotion transformée par l'œuvre, qu'il conçoit comme un «dispositif »: par différents procédés (le rythme, les matériaux, sonores, les procédés narratifs, les choix lexicaux), l'œuvre mobilise en nous des affects contraires, crée des attentes, exacerbe des contradictions...

Afin de rendre perceptibles la transparence de l'artefact et les schèmes sociaux d'utilisation que nous supposons déjà formés extérieurement à l'usager (Rabardel, 1995), nous proposons un rapprochement entre la structure du dispositif de formation de la plateforme Néopass@ction décrite précédemment, et celles d'œuvres plastiques ou littéraires, en tant que dispositifs et espaces, autant dynamiques que dialogiques; en tant qu'« espaces de travail et espaces de paroles » (Faïta, 1999).

Ouvrons les yeux sur ce thème 2 . On pourrait dire ici, qu'à l'instar de compositions picturales classiques, on y trouve un personnage de premier plan (ad monitor), Claire, Albane ou Guillemette par exemple qui, tout en participant à la scène, interpelle le spectateur et l'invite à regarder le fond, non pas d'un tableau, mais celui de son activité, créant ainsi une interface entre la condition d'externalité de l'utilisateur et son entrée progressive dans la situation (ici, de travail). À cela, on peut ajouter la composition dynamique que forment les visages et les gestes des enseignants, novices et expérimentés, présents sur les différents écrans, l'existence d'un croisement des gestes et des directions des regards qui, telles les peintures de la Renaissance, donnent à voir une sorte d'épaisseur du métier et laissent imaginer l'espace potentiel dans lequel il se déploie. Il ne s'agit pas de personnages statiques, de figures isolées, d'icônes froides du travail, mais bien d'acteurs donnant chair et vie à la dimension collective de leur métier. Ils figurent - à tous les sens du terme - la polyphonie du discours face aux situations de travail. Pour le dire à la manière d'Yves Clot (2010), ces gens ont leur « travail à cœur » et ne craignent 
pas de donner à voir et à entendre les controverses professionnelles qui les animent et permettent de maintenir la vitalité de leur métier.

Ainsi, ce ne sont pas des vignettes indépendantes les unes des autres que révèlent ces écrans (cf. Figure 1). À la manière d'un retable ou d'un polyptique - que l'on pouvait cacher ou dévoiler au rythme de l'année liturgique, en pliant la surface ou en déployant l'ensemble de l'œuvre -, il s'agit bien ici de pouvoir accéder à un panneau central et aux volets et prédelles qui l'entourent. Rappelons que les œuvres anciennes ici convoquées étaient notamment destinées à l'apprentissage de populations souvent peu ou pas alphabétisées, qui étaient amenées à s'interroger par le jeu des images entre elles sur la signification d'épisodes bibliques. En établissant le parallèle avec l'organisation de ces œuvres, on peut dire que la force des différents registres de figuration des écrans de la plateforme Néopass@ction, associés dans une structure d'ensemble, donne à voir et à interpréter des situations de travail réel que chacun est capable de lire selon son degré d'expérience. Nous rejoignons ici les thèses de «La psychologie de l'art » de Vygotski, qui appelle à considérer "la forme comme non statique», et pour cela invite à "prendre pour base de recherche le principe constructif, et comprendre la forme comme dynamique ». Ce qui signifie que "nous devrons étudier les facteurs qui composent l'œuvre d'art non pas dans leur structure statique, mais dans leur flux dynamique » (1925-2005, p. 305).

En considérant que ce sont des photographies qui s'offrent avant tout au regard de l'usager de la plateforme, on pourrait penser que le mouvement est arrêté. Or, ce medium a pour caractéristique d'arrêter le regard du spectateur sur un instant vécu par d'autres. Cartier-Bresson parlait d'«instant décisif», soulignant ainsi la singularité de ce qui est donné à voir et le caractère signifiant de cet instant par rapport à la situation. Mais, face à cet arrêt temporel et décontextualisé de l'activité d'autrui, matérialisé par l'image, le spectateur se doit de reconstruire l'instant d'avant ou d'imaginer celui d'après. En cela, la photographie "s'adresse » aux autres, convie au dialogisme et incite l'usager à activer l'extrait vidéo dont elle est issue pour comprendre l'activité dans laquelle elle s'inscrit.

Cette conception dynamique concernant les œuvres plastiques, se retrouve dans les travaux Jean Arnaud (2013), qui montrent que dans de nombreuses œuvres contemporaines, l'expérience matérielle et mentale de l'artiste pour construire un objet ou un dispositif plastique doit être relayée par l'expérience individuelle et singulière qu'en effectue le spectateur, afin que l'œuvre d'art prenne sens dans son lieu d'exposition. Selon cet auteur, c'est en termes d'interaction et de transaction avec le milieu de l'œuvre que se joue le rapport au tiers. Ainsi, les artistes qui utilisent de telles procédures « désamorcent les dérives de la pensée unique en demandant au spectateur d'être acteur ("spectateurs-acteurs") d'une construction critique » (ibid.).

\subsection{Néopass@ction, entre plateforme documentaire et espace critique : une ressource de métier}

45 Le même auteur soutient l'hypothèse d'un »tiers espace plastique " fondé sur l'interférence des perceptions et sur la confrontation de leur interprétation émotionnelle dans une continuité structurelle de l'œuvre. Cette conception du «Tiers» permettrait l'existence de différents niveaux de réalité dans la complexité, ce qui est le cas de beaucoup d'installations qui provoquent l'échange et le contact entre les «spectateurs». Il ne s'agit pas simplement de considérer l'interactivité sensorielle ou la multi- 
dimensionnalité de ces espaces, mais de les considérer comme des lieux plastiques qui instaurent une incertitude sur les limites entre l'espace interne de l'œuvre et l'espace réel, ou des lieux dans lesquels l'expérience ordinaire du vide et du plein doit s'infléchir et se relativiser. C'est en cela que ces espaces deviennent « critiques " (du verbe krinein : séparer, choisir, décider, passer au tamis) ; ils permettent de concevoir l'œuvre « comme lieu de mise en crise, d'interrogation radicale et d'analyse, permettant de trier et de différencier » (ibid.). Nous considérons que les espaces de Néopass@ction s'apparentent à certaines œuvres fondées sur l'interférence, donc sur la fréquentation de lieux ouverts, car spatialement a-normaux, sur le déplacement mental et sur la déstabilisation sensorielle d'un "sujet-spectateur "; ils participent ainsi à la création de tiers dans des espaces critiques.

Par ailleurs, au-delà de l'expérience spatio-temporelle de l'œuvre d'art ou de la plateforme de ressources, ces échanges se font par le biais du langage et du discours entre interlocuteurs dans des espaces tiers.

Selon Faïta (2013b), le rapport dialogique est caractérisé par son intertextualité reprenant l'idée de Bakhtine pour qui « tout texte se situe à la jonction de plusieurs textes dont il est à la fois la relecture, l'accentuation", fondant ainsi une théorie critique du sens... à l'intersection d'autres textes, existants ou possibles. L'auteur souligne que le rapport dialogique ne peut fonctionner sans les rapports logiques et sémantiques (donc linguistiques) constituant la base formelle des énoncés, sans pour autant s'y réduire. De notre point de vue, chacune des composantes ou «vignettes » composant les écrans de Néopass@ction constitue un énoncé. Faïta souligne que ces rapports formels doivent, pour devenir dialogiques, s'imprégner de sens, s'incarner; autrement dit, " entrer dans une autre sphère d'existence " (Bakhtine, 1998, p. 255). Le dialogisme est en permanence tributaire d'une "troisième voix » combinant les "voix » des autres, de ceux à qui les signes du discours ont aussi appartenu.

Il nous semble ici qu'un parallèle peut être établi entre ce qu'Arnaud définit comme « tiers espace " par rapport à l'expérience plastique et ce que Faïta qualifie de « troisième voix » (2013b). Ce dernier souligne que le " principe dialogique » nourrit des recherches et des interventions dans le champ social, intégrant les différentes dimensions des activités humaines à l'étude : des activités productives, de travail comme de création. Le dialogisme bakhtinien est fondé sur l'inaccompli de tout échange entre les hommes. Cette conscience de "l'inaccompli » est motrice pour certaines œuvres centrées sur la perception de l'espace, entre réel et fiction, qui sont « conçues à partir d'une conscience du vide comme lieu de la relation» (Arnaud, 2013). Ce vide, cette relation interstitielle, "peut caractériser toutes les œuvres demandant au spectateur de s'impliquer pour l'activer dans un lieu, ce qui est particulièrement déterminant lorsque les repères habituels y sont défaillants » (ibid.).

Le rapprochement entre divers domaines d'activité préoccupe également Yves Clot qui souligne la part constitutive d'irréalisé de l'activité, qu'elle soit référée au monde du travail où à la sphère artistique ; concernant la confrontation à l'œuvre d'art, il écrit :

«à la rencontre de l'irréalisé en nous, et en jouant de l'inaccompli en dehors de nous, l'art agence un devenir de l'inconscient. Il ne dérive pas d'un d'un inconscient déjà donné. Il le produit en le récréant » (Clot, 2006).

Par ailleurs, pour Vygotski : 
« la forme est en guerre avec le contenu, elle se bat avec lui, en triomphe, et c'est dans cette contradiction dialectique entre le contenu et la forme que se situe le sens psychologique de la réaction esthétique " (1925/2005).

Il s'agit donc de considérer des principes de réaction, d'action, et de création. pour la dialectique qu'elle initie entre les contenus et la forme, en appréhendant certaines caractéristiques narratives de formes artistiques, littéraires, fictionnelles, en regard de celles de Néopass@ction plus directement en prise avec le réel.

\subsection{Se raconter des histoires pour réagir}

51 Précisons immédiatement qu'il ne s'agit pas de comparer le thème 2 de Néopass@ction à un traditionnel manuel scolaire où les leçons s'enchaîneraient les unes après les autres, ou de l'assimiler directement à l'expérience sensible d'une œuvre d'art. Néanmoins, certains aspects de la structure de la plateforme peuvent être associés à celle du livre ou $\mathrm{du}$ récit visuel d'une œuvre plastique, et à leur organisation narrative en grands chapitres, thèmes, registres, annexes, postscriptum, illustrations... A l'instar d'un roman, on y retrouve certaines héroïnes (Guillemette, Claire, Albane) aux prises avec des situations difficiles, auxquelles on peut s'identifier; on peut alors les suivre dans un déroulé temporel, dans leurs contradictions, leurs empêchements, et finalement dans la dynamique de leur développement, puisque la plateforme les montre à divers stades de leur expérience professionnelle.

Concernant la construction discursive, reprenons un instant le parallèle que nous avons établi entre le fonctionnement des écrans de Néopass@ction et celui de certaines œuvres plastiques combinatoires.

53

Il est possible de rapprocher les procédures artistiques de feuilletage spatial des procédés littéraires de construction d'un récit multiple, fondé sur la superposition de plusieurs descriptions distinctes ou de plusieurs « voix » (tels que les ont mis en œuvre par exemple Simon 1971, 1986; Calvino 1979; Danielevski, 2002). Ces écrivains construisent leurs histoires par démultiplication des niveaux de narration. Si nous considérons la juxtaposition d'éléments textuels et d'extraits de «travail réel» dans Néopass@ction, notons qu'autant l'écrivain Claude Simon que le plasticien Robert Rauschenberg incluent à leurs œuvres des éléments factuels et documentaires directement prélevés dans la réalité (coupures de journaux, photos d'actualités sérigraphiées, affiches de rue, objets divers : couvre-lit, paravents, animaux empaillés...). Chez ces deux artistes, le récit fait surface par l'activation de diverses matières-durée en mouvement, et « le spectateur construit l'histoire en fonction des parcours qu'il établit entre les éléments confrontés » (Arnaud, 2014). Selon ses termes, Claude Simon utilise les mots en tant que « carrefour de sens » (1986, p. 28). Il ne s'agit pas de démarches qui consistent à s'approprier l'espace réel en y rapprochant divers objets, mais il est question d'espaces qui transfigurent ou métamorphosent les choses par altérations mutuelles, qui ne se construisent pas sur leur seule distribution dans un espace unitaire. L'une des figures marquantes de ce type de construction transposée au cinéma et au multimédia est Chris Marker; dans «Immemory » (1997), celui-ci propose au spectateur de construire son propre récit de manière aléatoire, en cliquant sur des écrans interactifs pour choisir les éléments visuels, sonores et textuels de son itinéraire. 

disposition dans Néopass@ction et les hypothèses de cheminement de pensée et d'action d'un utilisateur de cet artefact (cf. figure 2 et chapitre suivant), notons qu'à propos de son œuvre «Si par une nuit d'hiver un voyageur » (1979), Italo Calvino déclare qu'il «fait remonter trop d'histoires à la fois, pour que l'on sente autour du récit d'autres histoires, qu'il pourrait raconter, ou qu'il racontera peut-être [...], un espace rempli d'histoires où l'on peut, comme dans l'espace, se déplacer dans toutes les directions [...] de sorte qu'à partir de n'importe quel moment ou de n'importe quel endroit on rencontre toujours la même épaisseur de matière à raconter ".

Mais dans cette abondance de récits, par où commencer? Dans l'hommage que Paul Valéry rend à Proust (1957, p. 772), il affirme que la spécificité de son œuvre joue sur « la surabondance des connexions que la moindre image trouve si aisément dans la propre substance de l'auteur ", et que la valeur de l'œuvre de Proust tient à sa remarquable capacité à être ouverte à n'importe quelle page : «L'intérêt de ses ouvrages réside dans chaque fragment. On peut ouvrir le livre où l'on veut; sa vitalité ne dépend point de ce qui précède, et en quelque sorte de l'illusion acquise; elle tient à ce que l'on pourrait nommer l'activité propre du tissu même de son texte ».

Il nous apparaît que cette spécificité de lecture est également offerte aux usagers de Néopass@ction.

\subsection{Néopass@ction et le lien hypertexte / hyperimage}

57 À la manière des œuvres combinatoires ou interactives citées (plastiques ou littéraires), les écrans de Néopass@ction (180 extraits vidéo pour le seul thème 2) créent des liens entre documents et récit, aussi bien qu'entre réel et fiction, dans des espaces interstitiels. Il s'agit d'un espace composite plein d'histoires et de situations possibles, de discours entremêlés concernant l'activité de travail des professeurs. Nous postulons que cet empilement « d'activités sur l'activité » contribue à convertir l'expérience individuelle en une « monnaie collective qui (...) peut entrer en circulation sur une base plus large que la seule relation interpersonnelle»(Bruner, 2000, p. 114). Finalement, se raconter des histoires, "c'est jeter un pont entre ce qui est établi et ce qui est possible ", c'est une «recherche de compromis entre autonomie et engagement alimenté par un processus dialectique entre ce qui nous est familier et ce qui serait possible» (Simonet, 2005). Nous illustrons notre propos par l'hypothèse qui suit. 
Figure 2 : Hypothèse d'un cheminement de pensée et d'action d'un utilisateur de Néopass@ction, entrant sur le Thème 2.

Figure 2: Hypothetical course of thought and action of a "Néopass@ction" user having selected section 2

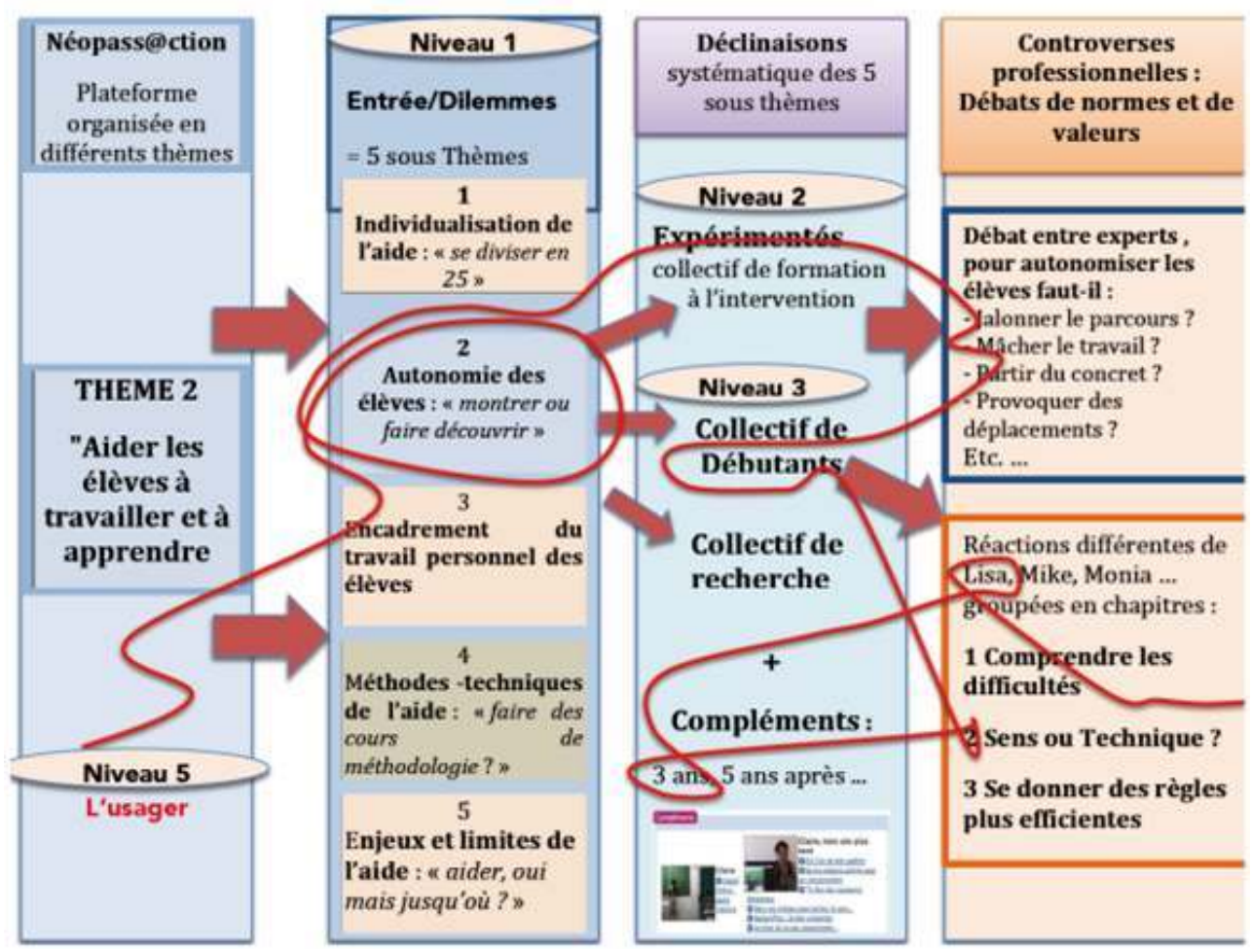

La figure ci-dessus illustre, à titre d'exemple, ce que pourrait être le cheminement de pensée et d'exploration d'un utilisateur de Néopass@ction, entrant sur le Thème 2 « Aider les élèves à travailler et à apprendre " de la plateforme. Nous avons figuré par des colonnes les différents registres de choix qui s'offrent à lui. Une fois ce thème 2 sélectionné, l'utilisateur est confronté à 5 sous-thèmes (colonne 2) correspondant aux entrées par les dilemmes professionnels.

Le tracé matérialise un choix hypothétique d'utilisateur qui, interpelé ici par «l'autonomie des élèves », se retrouve face à l'écran montré précédemment, où Claire se demande s'il vaut mieux « montrer » un exercice, une technique, une manière de faire ou d'apprendre à un élève en difficulté, ou « le lui faire découvrir ». Cette question n'a pas de réponse définitive en soi, le métier n'offrant aucune ressource " prête à penser et à agir " pour arbitrer ce dilemme; ici sont présents les débats de normes et de valeurs qu'il déclenche au sein des divers collectifs (colonne 3). L'utilisateur se retrouve donc à parcourir les multiples déclinaisons, interprétations individuelles et controverses collectives de ce sous-thème, incarnées par des professeurs débutants, expérimentés et des chercheurs... (colonne 4).

Même si « le discours n'est jamais le sous-titrage de l'action " (Faïta, 2012), l'utilisateur est guidé par une narration des gestes possibles de l'aide, constituée, entre autres choses, par les intitulés des séquences, des «mots du travail» directement issus des échanges verbaux entre les acteurs visibles de la plateforme ; il peut effectuer des aller-retour entre les différents registres d'images et de discours, arrêter les films, réécouter des réactions, relire des textes et utiliser ces façons de dire, en lien avec son propre degré d'expérience 
professionnelle et les observations issues des stages, pour construire, avec d'autres - en présentiel ou à distance -, son énonciation des situations de travail relatives à l'aide.

61 C'est à travers la parole du travail, portée par des gens du métier, à travers leur histoire singulière et collective, qu'il peut amorcer cette entrée progressive dans l'histoire du métier d'enseignement, dans ses aléas comme dans ses contraintes et ses victoires. Selon son expérience et sa maturité professionnelle, selon qu'il est seul ou en présence de pairs et/ou de formateurs, s'il est étudiant ou professeur débutant, il pourra explorer d'autres usages de la plateforme, imaginer d'autres investigations de la ressource.

Pour les concepteurs de cet artefact, l'un des enjeux de cette démarche est bien «de situer sur une même scène, dans un même cadre d'action, les logiques et les positions hétérogènes des opérateurs et des concepteurs pour faire œuvre commune " en assumant, durant la conception, que ses propriétés pourront être modifiées par les opérateurs à partir du développement de leur propre activité et compte tenu de leurs propres besoins, et en envisageant qu'une telle activité, de " conception continuée », en situation de travail, demande du temps (Béguin, 2013, p. 157).

\section{Conclusion}

63 Nous avons commenté la conception du thème 2 «aider les élèves à travailler et à apprendre » de Néopass@ction et les hypothèses instrumentales portées par cet artefact de formation. Nous nous sommes attachées à la matérialité des écrans de la plateforme pour montrer comment la forme et la structure dialogique de cet artefact autorisent les interactions, les «marges de manœuvre» et le rapport au milieu professionnel. Nous avons insisté sur le fait que la structure visuelle et lisible choisie pour didactiser cette ressource, reprend à son compte certains aspects du fonctionnement d'œuvres correspondant à des formes visuelles historiquement et culturellement fondées, et toujours réactualisées (le tableau, le livre, la B-D, les œuvres interactives et participatives, les espaces Internet et multimédias...). Ces «invariants culturels» (Béguin, 2013), ces codes d'organisation spatio-temporelle socialement construits sont familiers et permettent à l'usager d'accéder, sans se perdre, aux juxtapositions d'images, de textes, de films de Néopass@ction, et d'accéder partiellement à la complexité de l'activité d'autrui.

Mais nous avons peu abordé l'usage de ces ressources en formation professionnelle ; que peut-on en attendre?

Si l'on rejoint Bruner, cette structure dialogique, donnant accès aux caractères sociauxculturels et à l'histoire de l'activité enseignante, devrait permettre une entrée progressive dans une « culture de métier " par la confrontation de l'usager entre ce que, d'une part, il croit être ou savoir et, d'autre part, ce que donnent à entendre et percevoir les autres, car pour l'auteur:

« la culture tient sa vitalité de la dialectique entre des points de vue opposés, entre des récits qui s'affrontent. Le récit réinvente le présent et l'avenir comme il redéfinit les contours du passé » (Simonet 2005, à propos de Bruner 2002).

La plateforme Néopass@ction peut être considérée au sens de Meyerson (1948/1995) comme une œuvre que Bruner (1996a) caractérise comme « un mélange de connaissance et de technique codé de façon plus ou moins systématique»(p. 204). Cette œuvre composée de plusieurs artefacts (vignettes, photos, vidéo, textes...), eux-mêmes agencés différemment selon les thèmes et théories qui les sous-tendent, constituerait un rébus à 
réponses multiples (celui du travail) que les «lecteurs » ont à déchiffrer. Nous situons notre entreprise dans ce que Bruner nomme le monde des images qui « figent l'action et en font un récit. Lorsque l'action de l'homme parvient à s'exprimer en mots, ce n'est pas en terme de formule universelle et intemporelle, mais sous la forme d'histoires qui relatent les actions entreprises, les procédures suivies, etc. » (1996b, p. 196). Chacune des vignettes peut être considérée comme un élément du rébus que l'utilisateur peut activer et mettre en rapport avec d'autres vignettes, mais aussi avec d'autres acteurs ou protagonistes pour pouvoir re-penser le travail comme une activité dirigée. Peut-on alors considérer que cet artefact de formation, à l'instar des œuvres d'art, puisse « opérer sur son récepteur un effet de médiation, de réorganisation de la vie psychique consciente, par le jeu de procédures qui peuvent rester largement inconscientes»? (Bronckart, 1999, p. 29).

67 En considérant séparément chacun des thèmes aussi bien que l'ensemble de la plateforme de formation Néopass@ction, un certain nombre de thématiques se recoupent et mettent en évidence des significations communes, partagées par les expérimentés et les débutants. En revanche, certaines situations de travail révèlent des préoccupations de nature différente selon le degré d'expérience des uns et des autres. Nous avons pris le parti de conserver la trace de cette multiplicité de points de vue, car le métier enseignant n'est ni lisse, ni homogène. L'échange verbal et la communication que l'artefact génère, en intégrant la référence à l'action, permettent aux utilisateurs de thématiser l'échange et de piloter la compréhension de l'interlocuteur ; il permet ainsi d'aller à la rencontre de l'activité d'autrui pour orienter la sienne dans ce but (Faïta, 2012). Il ne s'agit évidemment pas de proposer des situations à imiter, mais il s'agit d'accéder à une communauté sociale réunie autour d'activités communes, d'entrer dans un ensemble de conventions partagées. «Ce partage nous inclut dans un monde de pratiques qui vont au-delà de l'individu, pratiques dont chaque opération dépend de ce qu'elle est communément distribuée. Ces pratiques sont comme un ensemble de rébus, reliés les uns aux autres", écrit Bruner (1996b, p. 192). Dans le même sens, Clot (1999) déclare que :

"l'expérience collective ne se transmet pas, elle dure et perdure sous la forme d'une évolution ininterrompue. Elle peut se perdre aussi. Mais quoi qu'il en soit, chacun ne reçoit pas en partage une expérience prête à l'usage. Il prend plutôt place dans le courant des activités et des gestes. Plus exactement, le geste personnel ne se construit que dans et contre ce courant en s'appropriant les rébus du genre ».

Pour de futurs professionnels, ou pour des non-initiés, la difficulté de s'approprier des gestes et de déchiffrer ces rébus professionnels, tient au fait que derrière le terme de «métier» se dissimulent des pans d'univers techniques, des recettes jalousement gardées, des routines, des "ficelles de métier» ancestrales. La conservation et la transmission de ce patrimoine induisent bien souvent des attitudes de repli, de défense, qui s'appuient sur des codes internes bloquant les innovations, et qui rendent illisibles les processus d'apprentissage. Paradoxalement, à la reconnaissance sociale d'un métier s'oppose l'opacité du langage permettant de l'apprendre et de le qualifier. Les termes employés pour le décrire restent en effet de l'ordre d'application de règles, de procédures d'exécution, mais ils laissent de côté la part d'initiative de l'acteur. Le plus souvent, « les autres » sont ainsi laissés à la surface du travail sans qu'ils accèdent à son essence (de la même façon que beaucoup de gens restent à la surface des œuvres d'art dans un rapport fascinatoire). Or, selon Cru (1995), ce qui fait la spécificité d'un "métier», ce sont justement les langues et les règles de métier; ces composantes sont reconnues et 
intériorisées par ceux qui travaillent ensemble. Cette conception de «métier » implique que l'on ne peut en saisir les contours que de l'intérieur.

De notre point de vue, le thème 2 de Néopass@ction et son entrée par les dilemmes de métier comme par ses codes langagiers et gestuels, ouvre la porte sur des registres de signes par lesquels est médiée l'activité enseignante, et qui restent généralement inaccessibles. Ces ressources sont appréhendées par l'usager par le biais de la parole du travail exprimée par la bouche de ceux qui agissent, mais aussi par les gestes, les postures, les conduites, les regards (...) des enseignants filmés, quels qu'ils soient. Les «mots du travail» sont également des formes historiques et culturelles dans lesquelles chacun se reconnaît, ils « nous parlent » et suscitent de l'émotion.

70 Au-delà de cette «interpellation» émotionnelle, de "cette technique sociale d'aiguisement des affects » par laquelle Vygotski (1925/2005) qualifie l'art, il ne suffira pas en formation de conduire à constater le rapport entre " ce qui est fait, ce qui est dit " et "ce qui est fait de ce qui est dit», mais bien de mettre ce rapport en discussion en regard des conditions réelles de travail et des prescriptions que découvriront peu à peu les étudiants en situation de stage.

Les écrans de Néopass@ction permettent de considérer dans l'échange avec autrui, l'enchevêtrement d'interprétations des façons d'agir et d'être au travail ; ils donnent accès au système de renormalisation des normes en vigueur du travail enseignant, en cherchant à s'emparer du rapport à ses mots et à ses gestes. Wolff nous dit que :

"plus les musiques sont complexes, plus "jouent" différents types et niveaux de causalité internes entrelacés. Et “jouer” doit ici s'entendre au double sens de jouer un rôle et de jouer les uns avec les autres en laissant du jeu entre eux pour l'inventivité » (2015, p. 71).

Il appartient au cadre de la formation professionnelle de souligner ces "règles du jeu » implicites de métier, le rôle des acteurs, et justement d'inciter au jeu... Enfin, pour poursuivre la "conception continuée dans l'usage" de cet artefact (Rabardel, \& Pastré, 2005), et donner suite au dialogue établi entre les personnes (collectifs de débutants, expérimentés, formateurs...) diversement situées dans le processus de conception-production-utilisation il faudra que les formateurs d'enseignants, institutionnels ou «de terrain ", acceptent d'entrer dans l'échange et, par là même, de renormaliser leur propre travail pour construire en formation une histoire collective et intergénérationnelle du métier enseignant.

\section{BIBLIOGRAPHIE}

Arnaud, J. (2013). Espaces critiques pour un monde complexe. Mémoire de synthèse en vue de l'Habilitation à Diriger des Recherches. Aix-Marseille Université. Novembre 2013.

Arnaud, J. (2014). L'espace feuilleté dans l'art moderne et contemporain. Aix-en-Provence : Publications de l'Université de Provence.

Bakhtine, M. (1998). Poétique de Dostoïevski. Paris : Seuil. 
Béguin, P. (2013). La conception des instruments comme processus dialogique d'apprentissages mutuels. In P. Falzon (Ed.) Ergonomie constructive (pp. 147-160). Paris : PUF.

Bronckart, J-P. (1999). La conscience comme « analyseur » des épistémologies de Vygotski et Piaget. In Y. Clot (Ed.) Avec Vygotski. Paris : La Dispute.

Bronckart, J.P. (2008). Un retour nécessaire sur la question du développement. In M. Brossard, \& J., Fijalkow (Eds.), Vygotski et les recherches en éducation et en didactiques (pp. 237-250). Pessac : Presses Univ. de Bordeaux.

Brossard, M. (1989). Espace discursif et activités cognitives : un apport de la théorie vygotskienne. Enfance, 42(1-2), 49-56, http://www.persee.fr/doc/ enfan_00137545_1989_num_42_1_1878

Brossard, M. (2012). Le Développement comme transformation par appropriation des œuvres et de la culture. In Y. Clot (Ed.). Vygotski maintenant (pp. 95-116). Paris : La Dispute.

Bruner, J. (1991).... Car la culture donne forme à l'esprit. De la révolution cognitive à la psychologie culturelle. Paris : Eshel.

Bruner, J. (1996a). Meyerson aujourd'hui : quelques réflexions sur la psychologie culturelle. In F. Parot (Ed.). Pour une psychologie historique. Écrits en hommage à Ignace Meyerson (pp. 193-207). Paris : PUF.

Bruner, J. (1996b). L'Éducation, entrée dans la culture. Les problèmes de l'école à la lumière de la psychologie culturelle. Paris : Retz.

Bruner, J. (2000). Culture et modes de pensée. Paris : Retz

Bruner, J. (2002). Pourquoi nous racontons-nous des histoires? Paris : Retz.

Calvino, I. (1979). Si par une nuit d'hiver un voyageur. Paris : Points / Seuil.

Clot, Y. (1999). Le geste est-il transmissible? Actes des $10^{e}$ Entretiens de la Villette. Apprendre autrement aujourd'hui.

Clot, Y. (2003). Vygotski : la conscience comme liaison. In L. Vygotski (Ed.), Conscience, inconscient, émotions (pp. 7-59). Paris : La Dispute.

Clot, Y. (2006). Lev S.Vygotski : le social dans la psychologie. Sciences Humaines ํo 170.

Clot, Y. (2010). Le travail à cœur. Paris : La Découverte.

Clot, Y. \& Faïta, D. (2000). Genres et styles en analyse du travail. Concepts et méthodes. Travailler, 4. 7-43.

Cole M, Hakkarainen P, \& Bredikyte M. (2010). Culture et apprentissage chez les jeunes enfants. In : Tremblay RE, Boivin M, Peters RDeV, eds. Encyclopédie sur le développement des jeunes enfants [sur Internet]. Montréal, Québec. http://www.enfant-encyclopedie.com/documents/ColeHakkarainen-BredikyteFRxp.pdf. Page consultée [16/06/2016]

Cru, D. (1995). Règles de métier, langue de métier : dimension symbolique au travail et démarche participative de prévention. Mémoire EPHE, Paris.

Danielewski, M Z. (2002). La maison des feuilles. Paris : Denoël.

Daniellou, F. (2004). L'ergonomie dans la conduite de projets de conception de système de travail. In P. Falzon (Ed.) Ergonomie. (pp. 359-374). Paris : PUF. 
Faïta, D. (1999). Analyse des situations de travail : de la parole au dialogue. In J. Richard-Zappela (Ed.). Espaces de travail, Espaces de paroles (pp. 127-137). Rouen : Publications de l'université de Rouen.

Faïta, D. (2007). L'image animée comme artefact dans le cadre méthodologique d'une analyse clinique de l'activité. Activités, 4 (2), 3-15, http://activites.revues.org/1660

Faïta, D. (2011). Théorie de l'activité langagière. In B. Maggi (Ed.) Interpréter l'agir. Un défi théorique . Paris : PUF.

Faïta, D. (2012). Transmettre ou agir pour transformer ? In Maggi B., \& Prot B. (Eds.), Développer le pouvoir d'apprendre : pour une critique de la transmission en éducation et en formation (pp. 6-15), http://amsacta.cib.unibo.it, Bologne : TAO Digital Library.

Faïta, D. (2013a). Langage et travail/ Linguaggio e lavoro. Débat sur la théorie de l'activité langagière. Bologne : TAO Digital Library.

Faïta, D. (2013b). Le Dialogisme. Laboreal, IX(1), http://laboreal.up.pt/

Faïta, D., \& Saujat, F. (2010). Développer l'activité des enseignants pour comprendre et transformer leur travail. In F. Saussez, \& F. Yvon, (Eds) Analyser l'activité enseignante : des outils méthodologiques et théoriques pour l'intervention et la Formation (pp. 41-71). Québec: Presses de l'Université de Laval.

Faïta, D., \& Viera, M. (2003). Réflexions méthodologiques sur l'autoconfrontation croisée. In R. Amigues, D. Faïta, \& M. Kerrhoubi (Eds.), Métier enseignant, organisation du travail et analyse de l'activité, SKHOLÊ, Hors-série no 1, 57-68, Aix-Marseille : IUFM.

Félix, C. (2014). De l'intervention-recherche à la production de ressources : quelle didactisation de l'activité pour la formation des enseignants? Recherche et formation, 75, 51-64.

Félix, C., Amigues R., \& Espinassy, L. (2014). Le rôle de l'observation par les professeurs de leur travail dans la construction « d'observables » pour la recherche. In J-F Marcel, P. Chaussecourte, L. Numa-Bocage (Eds.). De l'observation des pratiques enseignantes (pp. 52-62). CREN.

Félix, C., \& Espinassy, L. (2011).Production d'une ressource en ligne Programme Néopass@action : « aider les élèves à travailler». http://neo.ens-lyon.fr/neo

Félix, C., \& Espinassy, L. (2012). Du développement professionnel des enseignants débutants à une plateforme de formation « Néopass@ction »: Quelles articulations entre recherche et formation? $2^{e}$ colloque international de didactique professionnelle " Apprentissage et développement professionnel » 7-8 juin 2012 Nantes. http://didactiqueprofessionnelle.ning.com/page/colloque-2012-nantes

Félix, C., \& Saujat, F. (2008). L'aide au travail personnel des élèves entre déficit de prescriptions et « savoirs méthodologiques » : un double regard didactique et ergonomique. Les Dossiers des Sciences de l'Éducation, 20, 123-136.

Félix, C., Saujat, F., \& Combes, C. (2012). Des élèves en difficulté aux dispositifs d'aide : Une nouvelle organisation du travail enseignant. Recherches en Éducation, Hors-série (4), 19-30.

Flandin, S. (2015). Analyse de l'activité d'enseignants stagiaires du second degré en situation de vidéoformation autonome. Contribution à un programme de recherche technologique en formation. Thèse de doctorat. Université Blaise Pascal. Clermont-Ferrand.

François, F. (1993). Pratiques de l'oral. Paris : Nathan.

François, F. (1996). Communication, interaction, dialogue... Remarques et questions. Le Français aujourd'hui, 11, 11-23.

Leontiev, A. N. (1975/1984). Activité, conscience, personnalité. Moscou : Édition du Progrès. 
Marker, C. (1997). Immemory. Cd-rom de l'exposition. Paris : Centre Georges Pompidou Meyerson, I. (1948/1995). Les fonctions psychologiques et les œuvres. Paris : Albin Michel.

Prot, B. (2011). Apprentissage de la conduite et sécurité routière : Un dilemme de référence pour la conception d'un référentiel de diplôme d'enseignant. Activités, 8(2), 189-201, http:// activites.revues.org/2599

Rabardel, P. (1995). Les hommes et les technologies. Paris : Armand Colin.

Rabardel, P., \& Pastré, P. (2005). Modèles du sujet pour la conception : dialectiques, activités, développement. Toulouse : Octarès.

Ria, L., \& Leblanc, S. (2011). Conception de la plateforme de formation Néopass@ction à partir d'un observatoire de l'activité des enseignants débutants : enjeux et processus. Activités, 8(2), 150-172, http://activites.revues.org/2618

Ria, L., \& Vidal-Gomel, Ch. (2014). Conception d'environnements de formation : une entrée par l'analyse de l'activité. Activités, 11(2). http://activites.revues.org/964

Rickenmann, R. (2012). La construction sociale de l'émotion esthétique : dimensions transactionnelles des rapports à l'objet culturel en classe. In J.-Ch. Chabanne, M. Parayre, \& E. Villagordo (Eds.), La Rencontre avec l'œuvre. Éprouver, pratiquer, enseigner les arts et la culture (pp. 317-336). Paris : L'Harmattan.

Rimé, B. (2005). Le partage social des émotions. Paris : PUF

Saujat, F. (2010). Travail, formation et développement des professionnels de l'éducation : voies de recherche en sciences de l'éducation. Note de synthèse pour l'Habilitation à Diriger des Recherches (non publiée). Université de Provence.

Saussez, F. (2014). Une entrée activité dans la conception d'environnements de formation pour sortir d'une vision fonctionnaliste de la formation, un essai de conclusion. Activités, 11(2), 188-200. http://activites.revues.org/969

Saussez, F. (2014). Analyse de l'activité des enseignants débutants et vidéoformation. Recherche et formation, $75 \mid 2014$

Sève, L. (2002). Quelles contradictions ? À propos de Piaget, Vygotski et Marx. In Y. Clot (Ed.),

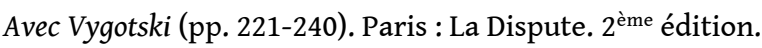

Simon, C. (1971). Les corps conducteurs. Paris : Minuit.

Simon, C. (1986). Discours de Stockholm. Paris : Minuit.

Simonet, P. (2005). J. Bruner. Pourquoi nous racontons-nous des histoires ? L'orientation scolaire et professionnelle [En ligne], 34/2 | 2005, mis en ligne le 28 septembre 2009, consulté le 23 mars 2013. URL : http://osp.revues.org/522

Valéry, P. (1957). Cuvres I, LP +. Paris : Gallimard / Pléiade.

Vygotski, L.S. (1933/1998). Théorie des émotions : étude historico-psychologique. Paris : L'Harmattan.

Vygotski, L.S. (1934/1997). Pensée et Langage. Paris : La Dispute

Vygotski, L.S. (1925/1994). Le problème de la conscience dans la psychologie du comportement. (Traduction de Françoise Sève), Société Française, 50, 35-47.

Vygotski, LS. (1925/2005). Psychologie de l'art. Paris : La Dispute. 
Wertsch, J. (1985). La médiation sémiotique de la vie mentale; L. V. Vygotski et M. M. Bakhtine. In B. Scheuwly \& J.-P. Bronckart (Eds.). Vygotski aujourd'hui (pp. 139-168). Neuchâtel : Delachaux et Niestlé

Wisner, A. (1995). Réflexions sur l'ergonomie. Toulouse : Octarès.

Wolff, F. (2015). Pourquoi la musique ? Paris : Fayard.

\section{NOTES}

1. D'autres thèmes de cette plateforme sont conçus par l'équipe ERGAPE selon le principe décrit ici (J-C Mouton : «faire classe en cours double » et « lycée professionnel »), et d'autres selon des ancrages théoriques relatifs au "cours d'action ", mais nous nous bornerons à ne parler que de celui dont on nous a confié la conception.

\section{RÉSUMÉS}

Nous interrogeons la forme et l'usage d'un artefact de formation destiné au métier enseignant proposant une "entrée activité » : la plateforme de ressources en ligne Néopass@ction, et plus particulièrement le thème «Aider les élèves à travailler et à apprendre " (Accès en ligne : http:// neo.ens-lyon.fr/neo) que nous avons conçu (Félix \& Espinassy, 2011). Nous soutenons l'hypothèse que les choix de conception, notamment une entrée par les «dilemmes professionnels ", ainsi que l'organisation formelle de ces ressources correspondant à des formes historico-culturelles préexistantes (artistiques, plastiques, littéraires...), favorisent une mise en débat de situations de travail comme moyen d'entrer progressivement dans le métier. Nous interrogeons la structure dialogique de cet artefact et les « hypothèses instrumentales » des opérateurs et des concepteurs.

We examine the design, the form and the intended usage of a teacher training artefact providing an "activity-oriented approach": the "Néopass@ction" online resource platform and particularly the section we authored entitled "Helping pupils to work and learn" (Félix, Espinassy, 2011). We argue that the design options, notably the "professional dilemma" approach, and the formal organization of the resources modelled on pre-existing historical-cultural forms (artistic, plastic, literary, etc.), encourage debate on work situations as a means of gradually integrating a profession. We examine the dialogical nature of the artefact and the "instrumental hypotheses" of both the users and the designers.

\section{INDEX}

Keywords : artefact design, professional training, teaching, professional dilemma, artistic forms, cultural invariants, dialogism, Néopass@ction

Mots-clés : conception artefact, formation professionnelle, enseignement, dilemmes professionnel, formes artistiques, invariants culturels, dialogisme, Néopass@ction 


\section{AUTEUR}

\section{LAURENCE ESPINASSY}

Aix Marseille Université, ENS Lyon, ADEF EA 4671, Equipe ERGAPE, 13248 Marseille

laurence.espinassy@univ-amu.fr 